\title{
Société Canadienne d'Études de la Renaissance / Canadian Society for Renaissance Studies
}

La réunion annuelle de la Société Canadienne d'Etudes de la Renaissance s'est tenue à la l'Université du Québec à Montréal du 28 au 30 mai 1980, dans le cadre du Congrès des Sociétés savantes.

La première séance, consacrée au théâtre français de la Renaissance, tenue conjointement avec l'Association des professeurs de français des universités canadiennes, et présidée par Michel Bareau (Université de l'Alberta), comprenait les communications suivantes: Claude Levy (Université Concordia), "La Cléopâtre de Shakespeare et celle de Jodelle"; Antoine Soare (Université Queen's), "Les Tragédies de l'assassinat"; René Ledwidge (Université Acadia), "La Tragédie Françoyse du bon Kanut, Roy de Dannemarch (1575)"; Jean Schneider (Université Concordia), "L'Enfant dans le théâtre tragique du XVIe siècle"; Max Vernet (Université Queen's), "L'Histoire tragique au service du Prince".

$\mathrm{Au}$ cours de la deuxième séance, présidée par L. Lieblein (Université McGill) et qui portait sur le théâtre anglais de la Renaissance, furent entendues les communications suivantes: James Black (Université de Calgary), "English Drama and the Renaissance Art of War"; Neil Carson (Université de Guelph), "Thomas Heywood: Apologist for the Popular Theatre"; Roger A. MacDonald (Université Saint Mary), "Ben Jonson and the Character of the Widow"; Ronald Huebert (Université Dalhousie), "The Drama of Infidelity (1603-1635)".

La troisième séance, consacrée au théâtre espagnol de la Renaissance, tenue conjointement avec l'Association canadienne des Hispanistes, et présidée par Roger Moore (Université Saint Thomas), comprenait les communications suivantes: Loes ForthergillPayne (Université de Calgary), "The Search for a Formula, Spanish Theatre between 1570 and 1590"; Dawn Spanish (Université Trent), "El concepto de la Fortuna en La vida y muerte de Herodes de Tirso de Molina"; Roger Moore, "Illusory Sense perception and the Normal Data of Experience in El magico prodigioso".

La quatrième séance, présidée par Claire Dolan (Université Laval), regroupait des communications libres: Maurice Lebel (Université Laval), "Josse Bade (1462-1535?), Jodocus Badius, éditeur et préfacier"; Gaston G. Allaire (Université de Moncton), "L'ésotérisme dans la musique de la Renaissance"; Douglas J. Wurtele (Université Carleton), "Spenser's Allegory in the mind".

La Contre-Réforme était le sujet de la cinquième et dernière séance, présidée par Harry McSorley (Collège Saint Michael, Université de Toronto). Les communications suivantes y furent présentées: Denis Janz (Université Loyola), “Cardinal Cajetan: 
A Thomist Reformer?"; Laurence Murphy (Université Saint Mary), "Gabriel Biel as Source of a Counter-Reformation Debate on the Effect of Original Sin on the Intellect"; Dennis McAuliffe (Collège Scarborough, Université de Toronto), "Evangelical Catholic Literature from Devotion to Poetry in Pre-Tridentine Italy". Le président ajouta quelques mots en guise de conclusion: "The Confutatio Pontificia of the Augsburg Confession as an Ecumenical Document".

Ces communications, dont plusieurs seront vraisemblablement publiées dans une revue savante, donnèrent lieu à des échanges intéressants, voire très animés, entre les conférenciers et les participants.

Les membres de la Société eurent l'occasion de fraterniser davantage lors du banquet et d'assister à la présentation d'une saynète: "A Mery Play Betwene Johan Johan the Husbande Tib His Wife, and Sir Johan the Preest", dirigée par Dena Goldberg, études anglaises, Université de Montréal.

Lors de la séance plénière, le président et la secrétaire-trésorière sortants, le directeur de la revue Renaissance and Reformation/Renaissance et Réforme ainsi que le président du comité de mises en candidature présentèrent leur rapport. L'assemblée procéda ensuite à l'élection du nouveau bureau de la Société.

CALUDE SUTTO, Université de Montréal 